\title{
ВЫЧИСЛИТЕЛЬНАЯ ТЕХНИКА
}

\section{СВОЙСТВА ФИЛЬТРА КАЛМАНА В ПЕРЕХОДНОМ РЕЖИМЕ}

\author{
А. Ю. КАПЛИН ${ }^{1}$, М. Г. СТЕПАНОВ ${ }^{1}$, А. Г. ЯРМОЛИЧ ${ }^{1,2}$ \\ ${ }^{1}$ ОАО „Радиоавионика“, 190005, Санкт-Петербург, Россия \\ E-mail:a.kaplin@radioavionica.ru \\ ${ }^{2}$ Балтийский государственный технический университет „ВОЕНМЕХ“ им. Д. Ф. Устинова, \\ 198005, Санкт-Петербург, Россия
}

\begin{abstract}
Рассматриваются условия достижения высокой точности фильтра Калмана в переходном режиме. Определяются соотношения между соответствующими значениями параметров уравнений состояния и измерения. Сформулировано несложное правило предварительного определения характера переходного процесса. На примере практически важной задачи калмановской оценки экспоненциально-коррелированного случайного процесса расчетным путем иллюстрируется применение предложенного правила при различных исходных данных.
\end{abstract}

Ключевые слова: переходный процесс, фильтр Калмана, установившийся режим фильтрации, точность оченивания

Фильтр Калмана традиционно используется в радиолокационных и навигационных комплексах, системах управления и связи, центрах обработки траекторных измерений [1-6] и др. Не ослабевает интерес к калмановской фильтрации и в настоящее время [7-10].

При использовании калмановских алгоритмов в конкретных комплексах и системах разработчики справедливо считают, что в рамках принятых моделей (уравнений) состояния и измерения фильтр Калмана обеспечивает минимальную (в среднеквадратическом смысле) ошибку оценивания по сравнению с любой другой линейной системой фильтрации вне зависимости от соотношения значений параметров этих моделей. Однако при этом разработчики зачастую пренебрегают характером переходного процесса или предполагают, что при оценке стационарных случайных процессов, описываемых уравнениями состояния и измерения с постоянными параметрами, зависимость ошибки оценивания каждого параметра от объема измерительной выборки представляет собой монотонно убывающую функцию, асимптотически приближающуюся к установившемуся значению. В результате не учитывается соотношение исходного уровня точности априорных данных (начальных условий) об оцениваемом процессе с уровнем точности его апостериорной оценки в установившемся режиме фильтрации. Примером такого подхода являются работы [7, 9].

В то же время существует ряд практических задач, где переходный режим фильтра Калмана не менее важен, чем установившийся. Продолжительность и точность переходного процесса, установившийся уровень точности фильтрации являются важными факторами в таких случаях, как наведение боевых элементов кассетных боеприпасов с активным ударным ядром (время стабилизации и прицеливания, как правило, меньше времени переходного режима), автоматическое сопровождение беспилотных летательных аппаратов квадрокоптерного типа или комплексирование систем спутниковой и автономной навигации в информационной системе бойца (спонтанное резкое изменение параметров уравнения состояния 
наблюдаемого объекта) и т.п. Предельным случаем является оценка нестационарных процессов, задаваемых уравнениями состояния и измерения с переменными параметрами. В этом случае фильтр Калмана также является линейной динамической системой с переменными параметрами, перманентно находящейся в режиме переходного процесса, характер которого определяет весь процесс фильтрации, и не учитывать который уже нельзя. На практике такая задача может иметь место при автоматическом сопровождении статически неустойчивых аэродинамических летательных аппаратов.

В разное время предпринимались отдельные попытки скорректировать переходный процесс фильтра Калмана, предлагались меры по его ускорению и повышению точности (см., например, $[1,11,12])$. Однако полученные результаты носили частный характер. Проанализируем возможные особенности фильтра Калмана и определим условия обеспечения высокой точности его переходного режима.

Цели работы - анализ условий, благоприятствующих высокой точности фильтра Калмана в переходном режиме; определение соотношений между соответствующими значениями параметров уравнений состояния и измерения; формулировка несложного правила предварительного определения характера переходного процесса.

Для анализа выберем базовый вариант фильтра $[13,14]$, являющийся основой большинства известных теоретических обобщений и практических модификаций процедуры калмановской фильтрации. Подлежащий оценке стационарный векторный случайный процесс $\mathbf{x}_{k}$ формируется в соответствии с уравнением состояния

$$
\mathbf{x}_{k+1}=\mathbf{\Phi} \mathbf{x}_{k}+\mathbf{w}_{k}, k=1,2, \ldots,
$$

где $\boldsymbol{\Phi}$ - постоянная переходная матрица; $\mathbf{w}_{k}$ - векторный дискретный нормально распределенный (гауссов) белый шум возмущений с нулевым математическим ожиданием и ковариационной матрицей $\mathbf{Q}, \mathbf{w}_{k} \sim N(\mathbf{O}, \mathbf{Q})$. Математическое ожидание и ковариационная матрица заданного начального условия $\mathbf{x}_{0}$ соответственно равны $\overline{\mathbf{x}}$ и $\mathbf{P}_{0}$.

Уравнение измерения имеет вид

$$
\mathbf{y}_{k}=\mathbf{H} \mathbf{x}_{k}+\mathbf{v}_{k}
$$

где $\mathbf{H}$ - матрица измерения; $\mathbf{v}_{k} \sim N(\mathbf{O}, \mathbf{R})$ - векторный шум измерений.

В этих условиях оптимальной по критерию минимума среднеквадратической ошибки несмещенного оценивания процесса $\mathbf{x}_{k}$ по измерениям $\mathbf{y}_{k}$ (минимума ковариационной матрицы ошибок $\mathbf{P}_{k}=M\left[\left(\mathbf{x}_{k}-\hat{\mathbf{x}}_{k}\right)\left(\mathbf{x}_{k}-\hat{\mathbf{x}}_{k}\right)^{T}\right]$, где $\hat{\mathbf{x}}_{k}$ - оценка вектора $\mathbf{x}_{k}, M[\bullet]$ - математическое ожидание) является линейная динамическая система, задаваемая соотношениями при начальных условиях $\overline{\mathbf{x}}$ и $\mathbf{P}_{0}$ :

$$
\begin{gathered}
\hat{\mathbf{x}}_{k}=\boldsymbol{\Phi} \hat{\mathbf{x}}_{k-1}+\mathbf{P}_{k} \mathbf{H}^{T} \mathbf{R}^{-1}\left(\mathbf{y}_{k}-\mathbf{H} \boldsymbol{\Phi} \hat{\mathbf{x}}_{k-1}\right), \\
\mathbf{P}_{k / k-1}=\boldsymbol{\Phi} \mathbf{P}_{k-1} \boldsymbol{\Phi}^{T}+\mathbf{Q} \\
\mathbf{P}_{k}=\left[\mathbf{P}_{k / k-1}^{-1}+\mathbf{H}^{T} \mathbf{R}^{-1} \mathbf{H}\right]^{-1}=\mathbf{P}_{k / k-1}-\mathbf{P}_{k / k-1} \mathbf{H}^{T}\left[\mathbf{H} \mathbf{P}_{k / k-1} \mathbf{H}^{T}+\mathbf{R}\right]^{-1} \mathbf{H} \mathbf{P}_{k / k-1} .
\end{gathered}
$$

Анализ показывает, что при определенных соотношениях между параметрами $\mathbf{Q}, \mathbf{R}$ и $\mathbf{P}_{0}$ в уравнениях (1), (2) переходный процесс и установившийся уровень точности фильтра Калмана могут различаться.

Обратимся к следующему практически важному, наглядному и простому случаю. Оценке подлежит скалярный случайный процесс, описываемый уравнением состояния [3]

$$
x_{k+1}=\Phi x_{k}+w_{k}
$$


где $\Phi=\exp \left(-\alpha_{x} T\right)-$ постоянный коэффициент; $w_{k}$ - дискретный белый гауссов шум с нулевым математическим ожиданием и дисперсией $Q=\sigma_{x}^{2}\left(1-\Phi^{2}\right), w_{k} \sim N(O, Q)$; начальное условие $x_{0} \sim N\left(\bar{x}, P_{0}\right) ; T=t_{k+1}-t_{k}$ - интервал дискретизации (шаг) по времени.

Рекуррентное соотношение (5) является для любых $T$ строгим дискретным аналогом стохастического дифференциального уравнения

$$
d x(t) / d t=-\alpha_{x} x(t)+\sqrt{2 \alpha_{x} \sigma_{x}^{2}} n(t)
$$

$(n(t)$ - стандартный белый гауссов шум с нулевым математическим ожиданием и единичной интенсивностью), описывающего непрерывный экспоненциально-коррелированный скалярный гауссов процесс $x(t)$ с шириной спектра $\alpha_{x}$ (обратно пропорциональна постоянной времени корреляции $T_{x}$ ) и стационарным значением дисперсии $\sigma_{x}^{2}$ [3].

Модель состояния (6) широко используется для описания статистической динамики случайной составляющей параметров движения маневрирующих объектов [2], при калмановской оценке корректируемых погрешностей датчиков инерциальных навигационных систем [7] и в других задачах.

Измерения проводятся в соответствии с уравнением

$$
y_{k}=x_{k}+v_{k},
$$

где $v_{k}$ - скалярный шум измерений, $v_{k} \sim N\left(0, \sigma_{v}^{2}=R\right)$.

В этом случае уравнения фильтра Калмана (3), (4) принимают следующий вид при начальных условиях $\hat{x}_{0}$ и $P_{0}$ :

$$
\begin{gathered}
\hat{x}_{k}=\Phi \hat{x}_{k-1}+\frac{P_{k}}{R}\left(y_{k}-\Phi \hat{x}_{k-1}\right), \\
P_{k / k-1}=\Phi^{2} P_{k-1}+Q, \\
P_{k}=\frac{P_{k / k-1} R}{P_{k / k-1}+R}=\frac{\left(\Phi^{2} P_{k-1}+Q\right) R}{\Phi^{2} P_{k-1}+Q+R} .
\end{gathered}
$$

Здесь $P_{k}=\sigma_{k}^{2}$ - текущее значение дисперсии ошибки оценки $\hat{x}_{k}, P_{k / k-1}$ - экстраполированное на шаг значение этой дисперсии, $P_{0}=\sigma_{0}^{2}-$ начальное значение дисперсии $P_{k}$, для $\hat{x}_{0}=\bar{x} \quad P_{0}=\sigma_{w}^{2}=Q$. Возможны другие варианты задания начального условия $\hat{x}_{0}$ и соответствующего значения дисперсии $P_{0}$.

Очевидно, что если с увеличением номера шага фильтрации $k$ дисперсия $P_{k}$ становится меньше начального значения $P_{0}$ и равномерно убывает (нисходящий переходный процесс), стремясь к установившемуся значению $P_{\infty}\left(P_{\infty}<P_{0}\right)$, фильтр Калмана обеспечивает повышение точности оценивания в обоих — переходном и установившемся - режимах. При таком характере переходного процесса применение фильтра целесообразно. Если переходный процесс сопровождается ростом $P_{k}$ и приводит к $P_{\infty}>P_{0}$ (возрастающий характер переходного процесса), вопрос о целесообразности калмановской фильтрации должен решаться в каждом конкретном случае исходя из целей и условий решаемой задачи.

Учитывая монотонный характер изменения $P_{k}$ с ростом $k$, для определения характера переходного процесса калмановского фильтра достаточно ограничиться сравнением величин $P_{1}$ и $P_{0}$ на первом шаге и проверить выполнение неравенства $P_{1}<P_{0}$, которому в соответствии с соотношением (8) при $k=1$ эквивалентно условие 


$$
Q\left(R-P_{0}\right)<\Phi^{2} P_{0}^{2}+P_{0} R\left(1-\Phi^{2}\right) .
$$

Выполнение неравенства (9) свидетельствует о нисходящем характере переходного процесса фильтра, невыполнение - о возрастающем.

Поскольку в (9) при всех возможных значениях параметров $\alpha_{x}$ и $T$ (см. уравнение (5)) $0<\Phi \leq 1$, правая часть в неравенстве (9) всегда положительна. Возможны два варианта выполнения (невыполнения) условия (9).

Вариант 1: $R \leq P_{0}$. Неравенство (9) выполняется при любых значениях дисперсии шума состояния $Q$ и любом способе задания начального условия $P_{0}$ (начального условия $\hat{x}_{0}$ в уравнении фильтра (7)), в том числе при $P_{0}=R$. Последнее означает, что в качестве начального условия $\hat{x}_{0}$ выбирается первое измерение $y_{1}$ с отсчетом последующих, начиная с $y_{2}$.

Bapиант 2: $R>P_{0}$. Из (9) видно, что при различных значениях $R, Q, P_{0}$ и Ф возможен как нисходящий (неравенство (9) выполняется), так и возрастающий характер переходного процесса (неравенство (9) нарушается). В последнем случае (9) приобретает вид $Q\left(R-P_{0}\right)>\Phi^{2} P_{0}^{2}+P_{0} R\left(1-\Phi^{2}\right)$, откуда легко получить пороговое значение $R=R_{\text {пор }}$ :

$$
R_{\text {пор }}=\frac{P_{0}\left(\Phi^{2} P_{0}+Q\right)}{\Phi^{2} P_{0}+Q-P_{0}},
$$

являющееся границей между областью повышения и областью понижения точности фильтрации в переходном режиме. Если при заданных условиями задачи значениях $Q, P_{0}$ и Ф (заданном $R_{\text {пор }}$ ) измерения $y_{k}$ проводятся с ошибкой $v_{k}$, имеющей дисперсию $R<R_{\text {пор }}$, переходный процесс имеет нисходящий характер; при $R>R_{\text {пор }}$ - восходящий. Заметим, что в отмеченном ранее практически важном случае $P_{0}=Q$ пороговое значение $R_{\text {пор }}=Q\left(\Phi^{2}+1\right) / \Phi^{2}$.

Возможен иной способ предварительного определения характера переходного процесса фильтра, заключающийся в сравнении начального значения дисперсии $P_{0}$ с установившимся значением $P_{\infty}$ дисперсии ошибки оценки $P_{k}$ при $k \rightarrow \infty$. При $P_{0}>P_{\infty}-$ нисходящий характер переходного процесса, при $P_{0}<P_{\infty}$ - возрастающий.

Значение $P_{\infty}$ можно получить, подставив в уравнение (8) единственное неизвестное $P_{k-1}=P_{k}=P_{\infty}$. В результате приходим к квадратному уравнению $\Phi^{2} P_{\infty}^{2}+\left[Q+\left(1-\Phi^{2}\right) R\right] P_{\infty}-$ $-Q R=0$, положительный корень которого есть искомое значение $P_{\infty}$ :

$$
P_{\infty}=\frac{-Q-\left(1-\Phi^{2}\right) R+\sqrt{\left[Q+\left(1-\Phi^{2}\right) R\right]^{2}+4 \Phi^{2} Q R}}{2 \Phi^{2}} .
$$

На практике предпочтителен более простой способ проверки выполнения неравенства (9) путем сравнения дисперсии ошибки измерений $R$ с $R_{\text {пор }}(10)$. В то же время более сложная процедура расчета дисперсии $P_{\infty}(11)$ позволяет установить конечную достижимую точность фильтрации.

Приведенные соотношения были использованы при расчете дисперсии ошибки оценивания $P_{k}$ как функции номера шага фильтрации $k$ для двух примеров. Первый (рис. 1$)$ иллюстрирует переходный процесс калмановской оценки случайной составляющей некоторого 
обобщенного параметра движения маневрирующего объекта. Типичными для подобных задач являются значения ширины спектра случайной составляющей $\alpha_{x}=1-3$ Гц и шага измерений $T=0,1-1$ с. В примере были выбраны $\alpha_{x}=1 \Gamma ц, T=0,1 \mathrm{c}, Q=0,362$. Расчеты проводились при фиксированной дисперсии случайной составляющей $\sigma_{x}^{2}=2$ для двух значений дисперсии ошибки измерений $R=4(a)$ и $6(\sigma)$. Использовались три варианта задания начального значения дисперсии $P_{0}$ : как одного из двух значений дисперсии $R(1)$, равного дисперсии шума состояния $Q$ (напомним, что $Q=\sigma_{x}^{2}\left[1-\exp \left(-2 \alpha_{x} T\right)\right]$ ) (2) и равного 0,1 (3). Последний вариант соответствует случаю высокоточных априорных данных о начальных условиях фильтрации.

a)

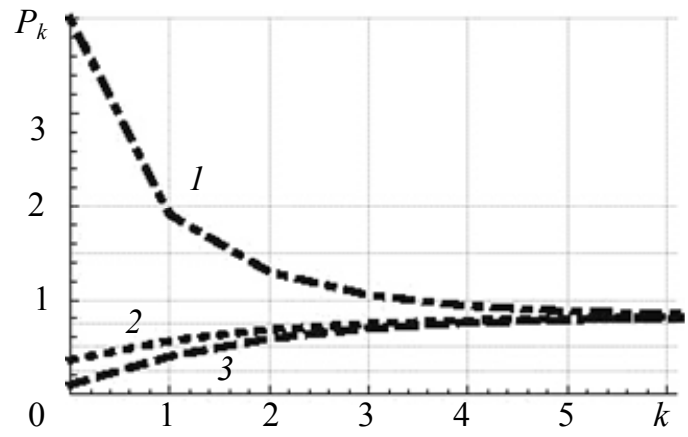

б)

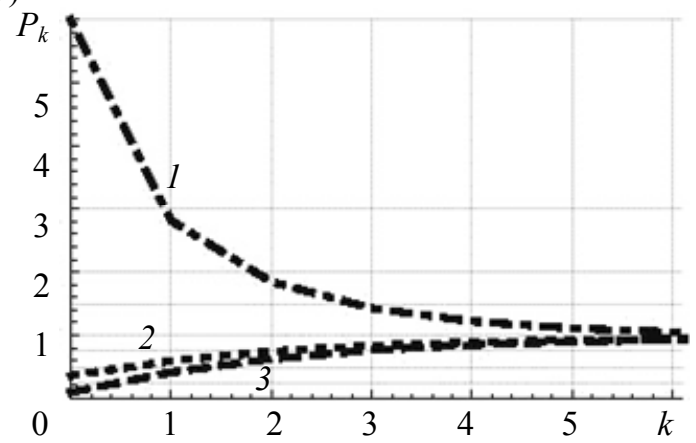

Puc. 1

Второй пример (рис. 2) соответствует задаче калмановской оценки погрешностей датчиков инерциальных навигационных систем. Типичными здесь являются значения $\alpha_{x}=5-10$ Гц, $T=0,01-0,05$ с. В расчетах использовались величины $\alpha_{x}=5$ Гц, $T=0,05$ с, $Q=0,786$. Остальные данные аналогичны первому примеру.

a)

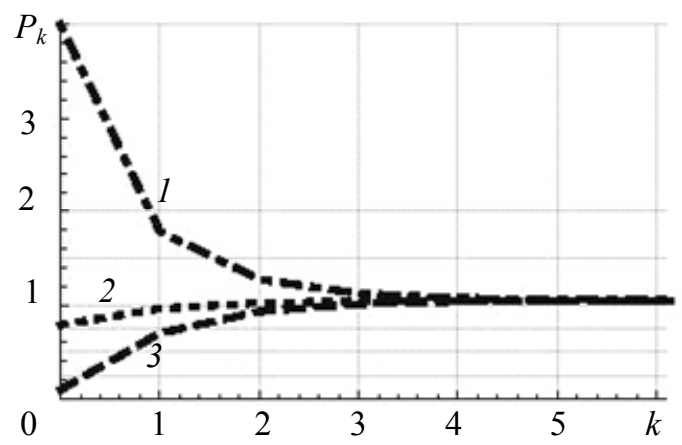

б)

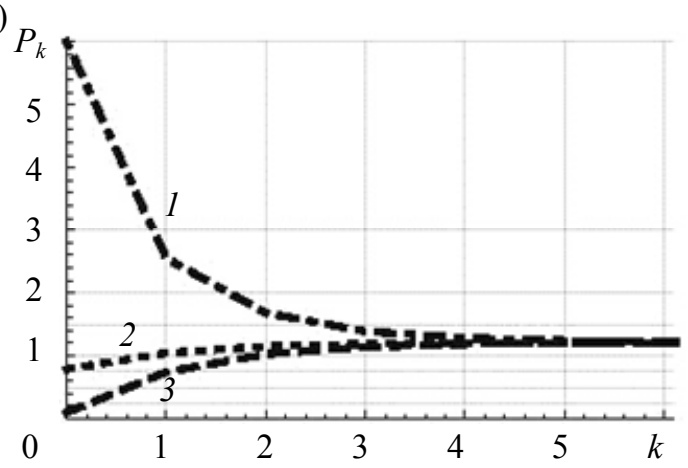

Puc. 2

Кривые на рис. 1, 2 подтверждают сделанный ранее вывод о зависимости характера переходного процесса фильтра Калмана (нисходящий или возрастающий) от сочетания исходных данных (параметров уравнений состояния и измерения), задаваемых условиями решаемой задачи. Продолжительность переходного процесса при этом может быть значительной.

В заключение важно отметить, что в настоящей статье речь идет о потенциальных точностных свойствах фильтра Калмана. Вопросы точности и сходимости (расходимости) фильтра, связанные с несоответствием реальной динамики случайных процессов принятым моделям (1), (2), погрешностями линеаризации при решении задач нелинейной фильтрации, вычислительными аспектами и другими факторами, здесь не рассматриваются. 


\section{СПИСОК ЛИТЕРАТУРЫ}

1. Кузьмин С. 3. Основы теории цифровой обработки радиолокационной информации. М.: Сов. радио, 1974. $432 \mathrm{c}$.

2. Шебшаевич В. С., Дмитриев П. П., Иванцевич Н. В. и др. Сетевые спутниковые радионавигационные системы. М.: Радио и связь, 1993. 408 с.

3. Ярльков М. С. Статистическая теория радионавигации. М.: Радио и связь, 1985. 344 с.

4. Кузовков Н. T., Сальчев О. С. Инерциальная навигация и оптимальная фильтрация. М.: Машиностроение, 1982. $216 \mathrm{c}$.

5. Сейдж Э., Мелс Дж. Теория оценивания и ее применение в связи и управлении. М.: Связь, 1976. 496 с.

6. Жданюк Б. Ф. Основы статистической обработки траекторных измерений. М.: Сов. радио, 1978. 384 с.

7. Иванов A. B. Навигационные системы подвижных наземных объектов. Алгоритмы обработки информации в угломерном канале // Радиотехника. 2013. № 4. С. 23-29.

8. Каплин А. Ю., Степанов М. Г. Использование автономной навигационной системы высокоточного позиционирования пешехода на местности // Информационно-управляющие системы. 2015. № 6. С. 86-92.

9. Каплин А. Ю., Степанов М. Г. Модель и алгоритм комплексной обработки информации азимутального канала пешеходной навигационной системы // Изв. вузов. Приборостроение. 2016. Т. 59, № 3. С. 181 - 188.

10. Советов В. М. Дискретный фильтр Калмана в задачах оптимального приема сигналов // Радиотехника. 2016. № 3. C. $43-49$.

11. Степанов М. Г. Использование редуцированных оценок для повышения устойчивости фильтра Калмана в переходном режиме // Изв. вузов МВ и ССО СССР. Приборостроение. 1984. Т. 27, № 1.

12. Степанов М. Г. Редуцированное оценивание вектора состояния линейной динамической системы // Изв. АН СССР. Техническая кибернетика. 1984. № 4.

13. Ли Р. Оптимальные оценки, определение характеристик и управление. М.: Наука, 1966. 176 с.

14. Браммер К., Зиффлинг Г. Фильтр Калмана-Бьюси. М.: Наука. Гл. ред. физ.-мат. лит., 1982. 200 с.

\section{Сведения об авторах}

Александр Юрьевич Каплин

Михаил Георгиевич Степанов

Алексей Григорьевич Ярмолич

Рекомендована

БГТУ „ВОЕНМЕХ“ им. Д. Ф. Устинова
Поступила в редакцию 01.09.16 г.

Ссылка для цитирования: Каплин А. Ю., Степанов М. Г., Ярмолич А. Г. Свойства фильтра Калмана в переходном режиме // Изв. вузов. Приборостроение. 2017. Т. 60, № 4. С. $311-317$.

\section{THE KALMAN FILTER PROPERTIES IN TRANSITION REGIME}

A. Yu. Kaplin ${ }^{1}$, M. G. Stepanov ${ }^{1}$, A. G. Yarmolich ${ }^{1,2}$

${ }^{1}$ Radioavionica JSC, 190005, St. Petersburg, Russia

E-mail: a.kaplin@radioavionica.ru

${ }^{2}$ D. F. Ustinov Baltic State Technical University (Voenmech), 198005, St. Petersburg, Russia

The conditions providing high accuracy of the Kalman filter in transition regime are considered. Relations between the respective values of parameters of state equations and measurement are determined. A simple rule for preliminary evaluation of the transition process nature is formulated. By the example of practically important problem of Kalman estimation of exponentially-correlated random process, application of the proposed rules is illustrated by presented calculations with different initial data. 
Keywords: transition regime, Kalman filter, steady-state filter mode, estimation accuracy

\section{Data on authors}

Alexander Yu. Kaplin _ PhD; Radioavionica JSC; E-mail: a.kaplin@radioavionica.ru

Mikhail G. Stepanov _ Pr. Sci., Professor; Radioavionica JSC; E-mail: smg099@mail.ru

Alexey G. Yarmolich - Radioavionica JSC; Leading Engineer; Post-Graduate Student; D. F. Ustinov Baltic State Technical University (Voenmech),

E-mail: note@iarmolich.com

For citation: Kaplin A. Yu., Stepanov M. G., Yarmolich A. G. The Kalman filter properties in transition regime // Journal of Instrument Engineering. 2017. Vol. 60, N 4. P. 311-317 (in Russian).

DOI: 10.17586/0021-3454-2017-60-4-311-317 